\title{
Percepção dos acadêmicos do Curso de Odontologia da UFCG sobre atuação do cirurgião-dentista no âmbito hospitalar
}

Perception of the UFCG undergraduate dental students on the dentist's performance in the hospital setting Percepción de los estudiantes de Odontología de la UFCG sobre el desempeño del dentista en el ámbito hospitalario

Nadjara de Freitas PEREIRA ${ }^{1}$

José Henrique de Araújo CRUZ $^{1}$

Luanna Abilio Diniz Melquíades de MEDEIROS ${ }^{2}$

Camila Helena Machado da Costa FIGUEIREDO²

Maria Angélica Satyro Gomes ALVES ${ }^{2}$

Elizandra Silva da PENHA ${ }^{2}$

Abrahão Alves de OLIVEIRA FILHO²

Gymenna Maria Tenório GUÊNES ${ }^{2}$

${ }^{1}$ Cirurgiã(o)-Dentista Curso de Graduação em Odontologia. Centro de Saúde e Tecnologia Rural, Universidade Federal de Campina Grande (UFCG) 58708-110 Patos - PB, Brasil

2 Professor(a) Doutor(a) do Curso de Graduação em Odontologia. Centro de Saúde e Tecnologia Rural,

Universidade Federal de Campina Grande (UFCG) 58708-110 Patos - PB, Brasil

\section{Resumo}

Introdução: A Odontologia Hospitalar é uma área de atuação da Odontologia que visa os cuidados das alterações bucais de pacientes internados em Unidade de Terapia Intensiva e enfermarias de hospitais. O conteúdo sobre a Odontologia Hospitalar tem se mostrado carente como parte integrante do currículo da graduação. Objetivo: analisar o conhecimento dos acadêmicos do Curso de Odontologia da Universidade Federal de Campina Grande sobre a atuação do cirurgião-dentista no contexto hospitalar. Métodos: Utilizou-se um questionário específico proposto por Jordão e Brito (2016) a respeito do conhecimento dos graduandos sobre odontologia hospitalar. Os dados foram organizados em planilhas do software Microsoft Excel e analisados sob a técnica descritiva. Resultados: A amostra foi composta por 258 acadêmicos do curso de odontologia, do total de acadêmicos, 92,24\% nunca teve uma experiência odontológica hospitalar. Todavia, 92,24\% reconhecem a importância de um cirurgião-dentista saber atender em um hospital. Na opinião dos estudantes, o conteúdo referente à Odontologia Hospitalar é carente no currículo da graduação $(42,63 \%)$. E a maioria dos alunos $(70,15 \%)$ não se sente capacitado para atuar junto a uma equipe multiprofissional no hospital. Conclusão: Pode-se constatar que os graduandos do curso de odontologia da UFCG têm ciência da importância da odontologia hospitalar, mas, admitem não ter um bom conhecimento sobre o assunto e não se sentem capacitados para atuar junto a uma equipe multiprofissional no hospital. Assim é necessário que durante a graduação o futuro cirurgião-dentista, seja incentivado e capacitado para o manejo do paciente em ambiente hospitalar.

Descritores: Assistência Odontológica; Odontologia; Unidades de Terapia Intensiva.

\section{Abstract}

Introduction: Hospital Dentistry is an area of practice in Dentistry that aims to care for oral changes in patients admitted to the Intensive Care Unit and hospital wards. Content on Hospital Dentistry has been found to be in need as an integral part of the undergraduate curriculum. Objective: to analyze the knowledge of the students of the Dentistry Course of the Federal University of Campina Grande on the performance of the dentist in the hospital context. Methods: We used a specific questionnaire proposed by Jordão and Brito (2016) regarding the knowledge of undergraduate students about hospital dentistry. The data were organized into Microsoft Excel spreadsheets and analyzed under the descriptive technique. Results: The sample consisted of 258 undergraduate dental students, of the total number of academics, $92.24 \%$ never had a hospital dental experience. However, 92.24\% recognize the importance of a dental surgeon knowing how to attend a hospital. In the opinion of the students, the content related to Hospital Dentistry is lacking in the undergraduate curriculum (42.63\%). And most of the students $(70.15 \%)$ do not feel able to work together with a multiprofessional team in the hospital. Conclusion: It can be observed that graduates of the UFCG dentistry course are aware of the importance of hospital dentistry, but admit that they do not have a good knowledge of the subject and do not feel able to work together with a multiprofessional team in the hospital. Thus, it is necessary that during graduation the future dental surgeon be encouraged and qualified to manage the patient in a hospital environment.

Descriptors: Dental Care; Dentistry; Intensive Care Units.

\section{Resumen}

Introducción: La Odontología Hospitalaria es un área de actuación de la Odontología que busca el cuidado de las alteraciones bucales de pacientes internados en Unidad de Terapia Intensiva y enfermerías de hospitales. El contenido sobre la Odontología Hospitalaria se ha mostrado carente como parte integrante del currículo de la graduación. Objetivo: analizar el conocimiento de los académicos del Curso de Odontología de la Universidad Federal de Campina Grande sobre la actuación del cirujanodentista en el contexto hospitalario. Métodos: Se utilizó un cuestionario específico propuesto por Jordania y Brito (2016) acerca del conocimiento de los graduandos sobre odontología hospitalaria. Los datos fueron organizados en hojas de cálculo del software de Microsoft Excel y se analizaron bajo la técnica descriptiva. Resultados: La muestra fue compuesta por 258 académicos del curso de odontología, del total de académicos, el 92,24\% nunca tuvo una experiencia odontológica hospitalaria. Sin embargo, el 92,24\% reconoce la importancia de un cirujano-dentista para atender en un hospital. En la opinión de los estudiantes, el contenido referente a la Odontología Hospitalaria es carente en el currículo de la graduación (42,63\%). Y la mayoría de los alumnos $(70,15 \%)$ no se siente capacitado para actuar junto a un equipo multiprofesional en el hospital. Conclusión: Se puede constatar que los graduandos del curso de odontología de la UFCG tienen ciencia de la importancia de la odontología hospitalaria, pero, admite no tener un buen conocimiento sobre el tema y no se sienten capacitados para actuar junto a un equipo multiprofesional en el hospital. Así es necesario que durante la graduación el futuro cirujano-dentista, sea incentivado y capacitado para el manejo del paciente en ambiente hospitalario.

Descriptores: Atención Odontológica; Odontología; Unidades de Cuidados Intensivos.

INTRODUÇÃO

A Odontologia Hospitalar $(\mathrm{OH})$ é uma área da Odontologia que atua em indivíduos que necessitem de atendimento em ambiente hospitalar, ou em assistência domiciliar e tem como objetivos: promoção da saúde, prevenção, diagnóstico e tratamento de doenças orofaciais, manifestações bucais de doenças sistêmicas ou decorrentes de seus respectivos tratamentos ${ }^{1}$. 
No Brasil, a Odontologia Hospitalar foi legitimada em 2004 com a criação da Associação Brasileira de Odontologia Hospitalar ${ }^{2}$. No ano de 2008, o Projeto de Lei № 2.776, que esta em tramitação no Senado Federal, estabelece a obrigatoriedade da presença de profissionais de Odontologia na UTI e enfermarias, portanto, os usuários internados nas enfermarias e UTIs deverão receber assistência odontológica ${ }^{3}$.

O tratamento integral do paciente é fundamental para que a equipe multidisciplinar atue de forma eficiente e segura, permitindo melhor desempenho no compromisso de melhora do quadro clínico do paciente internado ${ }^{4}$.

Os microrganismos que colonizam as superfícies da cavidade bucal podem ser disseminados para outras regiões do organismo através de procedimentos hospitalares de rotina na UTI, como no caso da entubação sob ventilação mecânica, que pode transportar bactérias presentes na cavidade bucal e da orofaringe até os pulmões, contribuindo para o surgimento da pneumonia nosocomial ${ }^{5}$.

As pneumonias nosocomiais são, de forma geral, as infecções de maior importância que podem acometer os pacientes hospitalizados ${ }^{6}$.

As doenças infecciosas na cavidade bucal estão intimamente relacionadas com alterações na resposta imunológica, falta de higiene oral, desnutrição severa, tabagismo, alcoolismo e diabetes, os quais podem aumentar a susceptibilidade ao desenvolvimento de gengivite, periodontite e doenças respiratórias ${ }^{7-9}$.

Um estudo realizado por Wayama et.al ${ }^{10}$ aponta que o conteúdo referente à Odontologia Hospitalar é carente no currículo da graduação. Em alguns casos, este tema é abordado apenas em nível de especialização ${ }^{11}$. O futuro profissional da área da saúde, o aluno graduando, durante a sua formação acadêmica, precisa ser incentivado e preparado para o manejo do paciente em âmbito hospitalar ${ }^{10}$.

O presente estudo busca avaliar o conhecimento dos acadêmicos do curso de Odontologia da Universidade Federal de Campina Grande (UFCG) campus Patos, matriculados do primeiro ao décimo período, sobre a odontologia hospitalar.

MATERIAL E MÉTODO

Foi realizado um estudo do tipo transversal, observacional, quantitativo com abordagem indutiva e procedimento comparativo, descritivo, adotando como estratégia de coleta de dados o questionário específico que avalia o conhecimento dos graduandos acerca da Odontologia hospitalar proposto por Jordão e Brito ${ }^{11}(2016)$. Esse questionário abordava questões como: já teve alguma experiência odontológica em um hospital?Tem interesse em vivenciar uma experiência odontológica em um hospital? Você se sente capacitado para atuar junto a uma equipe multiprofissional de um hospital? Entre outras. O universo foi composto por 373 alunos a partir do primeiro $\left(1^{\circ}\right)$ período, regularmente matriculados no Curso de Odontologia da Universidade Federal de Campina Grande campus de Patos.

A amostra foi constituída por 258 participantes. Para a participação dos graduandos nessa pesquisa foram considerados como critérios de inclusão: Ser estudante de odontologia da Universidade Federal de Campina Grande, campus de Patos; Estar regularmente matriculado do $1^{\circ}$ ao $10^{\circ}$ período; Autorização de participação da pesquisa por meio do Termo de Consentimento Livre e Esclarecido (TCLE) assinado. Foram excluídos da pesquisa os graduandos que apresentaram uma ou mais das seguintes características: Não estar presente na Sala de aula e/ou Clínica Escola de Odontologia da UFCG no dia da coleta.

Após coletados, os dados foram organizados em planilhas do software Microsoft Excel e analisados sob a técnica descritiva. $O$ projeto de pesquisa foi enviado para o Comitê de Ética em Pesquisa com Seres Humanos da Faculdade Integrada de Patos (FIP), e aprovado sob o CAAE: 05293618.3.0000.5181.

MATERIAL E MÉTODO

Foram aplicados 258 questionários respondidos por alunos de Odontologia da UFCG do primeiro ao décimo período. Dos estudantes que participaram da pesquisa, 62\% (160) eram do sexo feminino e 38\% (98) do sexo masculino (Tabela 1). Quanto a idade dos participantes, a faixa etária predominante foi de 17 a 23 (Tabela 2).

Quando questionados sobre já ter tido alguma experiência odontológica em um hospital, $92,24 \%$ (238) do total dos acadêmicos (258) nunca tiveram essa experiência (Tabela 3 ), e quando questionados sobre ter interesse em vivenciar alguma experiência odontológica hospitalar 92,24\% (238) acharam importante o cirurgião dentista saber atender em um hospital (Tabela 4). Quando questionados qual especialidade odontológica está mais capacitada para atender em um ambiente 
hospitalar, a CBMF 32,27\% (221) foi à especialidade mais lembrada pelos alunos dentre as opções fornecidas. Essa questão admitia uma ou mais alternativas (Tabela 5).

Tabela 1. Distribuição da amostra segundo o sexo.

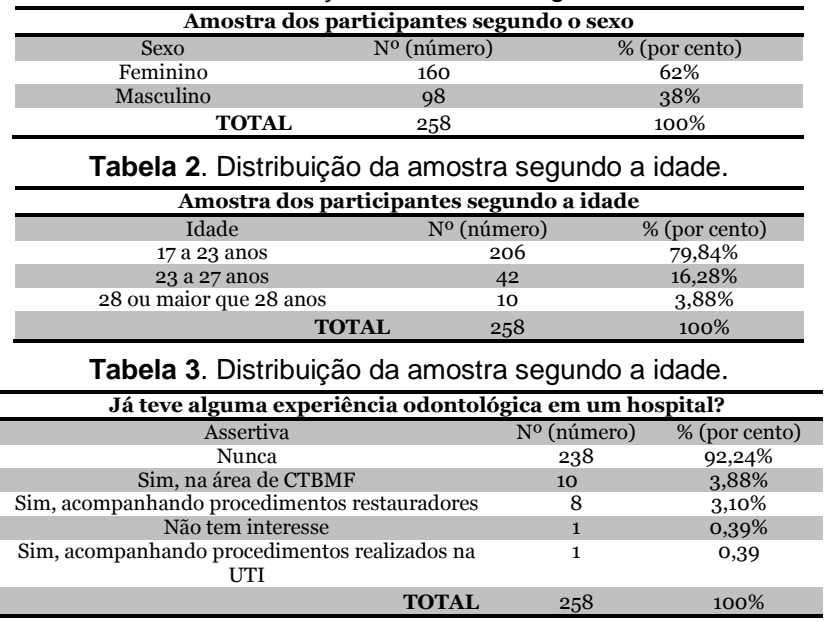

Tabela 4.Interesse em vivenciar alguma experiência odontológica no hospital.

\begin{tabular}{|c|c|c|}
\hline Tem interesse em vivenciar alguma expe & lontológ & \\
\hline Assertiva & $\overline{\mathrm{N}^{\circ} \text { (número) }}$ & $\%$ (por cento) \\
\hline $\begin{array}{l}\text { Acha importante um CD saber atender em um } \\
\text { hospital }\end{array}$ & 238 & $92,24 \%$ \\
\hline Acha que o hospital é somente para especialistas & 7 & \\
\hline Não acha necessário & 6 & $2,33 \%$ \\
\hline Não tem opinião á respeito & 4 & $1,56 \%$ \\
\hline Não gosta de hospitais & 3 & $1,16 \%$ \\
\hline Apenas por curlosidade, pois nao acha necessario & 258 & $0,00 \%$ \\
\hline
\end{tabular}

Tabela 5. Especialidade odontológica mais capacitada para atender no ambiente hospitalar na opinião dos alunos

\begin{tabular}{|c|c|c|}
\hline \multicolumn{3}{|c|}{$\begin{array}{c}\text { Qual a especialidade odontológica está mais capacitada para atender em } \\
\text { um ambiente hospitalar? }\end{array}$} \\
\hline Assertiva & $\mathrm{N}^{\circ}$ (número) & $\%$ (por cento) \\
\hline Cirurgia bucomaxilofacial & 221 & \\
\hline Estomatologia & 130 & $18,97 \%$ \\
\hline Odontologia para pacientes especiais & 108 & $15,77 \%$ \\
\hline Patologia & & \\
\hline Saúde coletiva & 84 & $\begin{array}{l}15,03 \% \\
12,27 \%\end{array}$ \\
\hline Implan & 21 & $\%$ \\
\hline Dentistica & 18 & $2,63 \%$ \\
\hline & 685 & $100 \%$ \\
\hline
\end{tabular}

Do ponto de vista de $42,63 \%$ (110) dos alunos que responderam ao estudo, a Odontologia Hospitalar é um conteúdo carente no currículo da graduação (Tabela 6 ).

Tabela 6. Odontologia hospitalar como parte integrante do currículo da graduação

\begin{tabular}{|ccc|}
\hline \multicolumn{3}{c|}{ Odontologia hospitalar como parte integrante do currículo da graduação } \\
\hline $\begin{array}{c}\text { Assertiva } \\
\text { Carente,apesar de já ter ouvido falar sobre o } \\
\text { conteúdo }\end{array}$ & 110 & $42,63 \%$ \\
$\quad \begin{array}{c}\text { Totalmente carente } \\
\text { (número) }\end{array}$ & 55 & $21,32 \%$ \\
\hline $\begin{array}{c}\text { Bom,apesar de ser fragmentado em outras } \\
\text { disciplinas }\end{array}$ & 32 & $12,40 \%$ \\
\hline $\begin{array}{c}\text { Não sabe,pois não conhece todo conteúdo da } \\
\text { faculdade }\end{array}$ & 32 & $12,40 \%$ \\
\hline Não sabe avaliar se há conteúdo no currículo & 29 & $11,25 \%$ \\
\hline$\quad$ TOTAL & 258 & $100 \%$ \\
\hline
\end{tabular}

Em relação ao componente curricular que deveria abordar o conteúdo referente à Odontologia Hospitalar, a Traumatologia com $16,67 \%$ (136) foi a opção mais lembrada pelos estudantes. Essa questão admitia uma ou mais alternativas (Tabela 7). Quando questionados sobre o que esperariam caso o conteúdo sobre a $\mathrm{OH}$ fosse incluído no currículo do curso, $45,35 \%$ (117) dos acadêmicos responderam que estar capacitado para atuar em um hospital quando estivessem formados (Tabela 8).
Tabela 7. Componente curricular que deveria abordar o conteúdo referente à $\mathrm{OH}$ na opinião dos acadêmicos.

\begin{tabular}{|c|c|c|}
\hline \multicolumn{3}{|c|}{$\begin{array}{l}\text { Em qual componente curricular deveria ser abordada a Odontologia } \\
\text { hospitalar? }\end{array}$} \\
\hline Assertiva & $\mathrm{N}^{\circ}$ (número) & $\%$ (por cento) \\
\hline Traumatologia Bucomaxilofacial & 136 & $16,67 \%$ \\
\hline Cirurgia Bucomaxilofac & 126 & $15,45 \%$ \\
\hline Saúde coletiva e odontologia preventiva & 97 & $11,90 \%$ \\
\hline Estomatologia & 86 & $10,53 \%$ \\
\hline Em uma disciplina eletiva & 86 & $10,53 \%$ \\
\hline Patologia bucal & 76 & $9,31 \%$ \\
\hline Terapêutica e Anestesio & 69 & $8,45 \%$ \\
\hline Biossegurança & 65 & $7,97 \%$ \\
\hline $\begin{array}{c}\text { Metabolismo humano e bases terapêuticas para } \\
\text { Odontologia }\end{array}$ & 46 & $5,64 \%$ \\
\hline Odontolo & 22 & $2,70 \%$ \\
\hline Outra & & $0,85 \%$ \\
\hline TOTAL & 816 & $100 \%$ \\
\hline
\end{tabular}

Tabela 8. O que o estudante esperaria caso o conteúdo referente à $\mathrm{OH}$ fosse incluído no currículo do curso

\begin{tabular}{|c|c|c|}
\hline \multicolumn{3}{|c|}{$\begin{array}{l}\text { O que esperaria caso o conteúdo sobre a odontologia hospitalar fosse } \\
\text { incluída no currículo do curso? }\end{array}$} \\
\hline Assertiva & $\begin{array}{c}\mathrm{N}^{\circ} \\
\text { (número) }\end{array}$ & $\begin{array}{l}\% \text { (por } \\
\text { cento) }\end{array}$ \\
\hline $\begin{array}{l}\text { Estar capacitado para atuar em um hospital quando } \\
\text { estivesse formado clínico geral. }\end{array}$ & 117 & $45,35 \%$ \\
\hline $\begin{array}{l}\text { Acompanhar um atendimento odontológico enquanto } \\
\text { aluno de graduação }\end{array}$ & 88 & $34,10 \%$ \\
\hline $\begin{array}{l}\text { Saber como um cirurgião dentista poderia atuar em um } \\
\text { hospital e como deveria se comportar }\end{array}$ & 53 & $20,55 \%$ \\
\hline TOTAL & 258 & $100 \%$ \\
\hline
\end{tabular}

Quando questionados se sentiam-se capacitados para trabalhar junto a uma equipe médica e/ou multiprofissional em um hospital, $70,15 \%$ (181) dos alunos responderam que não(Tabela 9).

Tabela 9. Opinião dos acadêmicos sobre sua capacidade de atuar em âmbito hospitalar

\begin{tabular}{|c|c|c|}
\hline \multicolumn{3}{|c|}{$\begin{array}{c}\text { Você se sente capacitado para trabalhar junto a uma equipe médica ou } \\
\text { multiprofissional em um hospital? }\end{array}$} \\
\hline Assertiva & $\mathrm{N}^{\circ}$ (número) & \% (por cento) \\
\hline Não & 181 & $70,15 \%$ \\
\hline Sim & 39 & $15,12 \%$ \\
\hline Não acha necessário & 0 & $0,00 \%$ \\
\hline $\begin{array}{c}\text { Nunca pensou na possibilidade de trabalhar junto a } \\
\text { uma equipe médica }\end{array}$ & 38 & $14,73 \%$ \\
\hline TOTAL & 258 & $100 \%$ \\
\hline
\end{tabular}

Sobre a presença de um cirurgiãodentista na equipe multiprofissional da Unidade de Terapia Intensiva, 54,65\% (141) dos estudantes acham importante na prevenção de infecções e outras doenças (Tabela 10).

Tabela 10. Presença do cirurgião-dentista na equipe multiprofissional da UTI

Sobre a presença de um cirurgião-dentista na equipe multiprofissional da UTI

\begin{tabular}{|c|c|c|}
\hline Assertiva & $\mathrm{N}^{\circ}$ (número) & $\%$ (por cento) \\
\hline $\begin{array}{c}\text { É importante na prevenção de infecções e outras } \\
\text { doencas }\end{array}$ & & \\
\hline $\begin{array}{l}\text { É importante para manter a saúde da cavidade bucal } \\
\text { dos pacientes }\end{array}$ & 108 & $41,86 \%$ \\
\hline $\begin{array}{l}\text { Os pacientes estão fora de condições de uma atuação do } \\
\text { cirurgiâo-dentista }\end{array}$ & 5 & $1,93 \%$ \\
\hline Não há necessidade de um cirurgião dentista na UTI & & $1,56 \%$ \\
\hline
\end{tabular}

Quando questionados se a presença do cirurgião-dentista na equipe multiprofissional poderia melhorar o quadro clínico geral dos pacientes, 96,9\% (250) dos acadêmicos responderam que sim, a assistência do dentista pode realizar a melhora do quadro clínico geral do paciente hospitalizado (Tabela 11). Por último, os estudantes responderam de que maneira a presença do cirurgião-dentista em um ambiente hospitalar poderia ser benéfica. Essa questão admitia uma ou mais alternativas. Dos estudantes que responderam a pesquisa $23,18 \%$ (198) afirmam que a presença do cirurgião-dentista em um ambiente hospitalar 
pode ser benéfica no tratamento de traumas faciais (Tabela 12).

Tabela 11. Opinião dos acadêmicos sobre se a presença do $C D$ na equipe multiprofissional pode melhorar o quadro clínico geral dos pacientes.

\begin{tabular}{|c|c|c|}
\hline \multicolumn{3}{|c|}{$\begin{array}{c}\text { A presença do cirurgião-dentista na equipe multiprofissional pode } \\
\text { melhorar o quadro clínico geral dos pacientes? }\end{array}$} \\
\hline Assertiva & $\mathrm{N}^{0}$ (número) & $\%$ (por cento) \\
\hline Sim & 250 & $96,9 \%$ \\
\hline Nunca pensei a respeito & 8 & $3,1 \%$ \\
\hline Não & o & $0,00 \%$ \\
\hline TOTAL & 258 & $100 \%$ \\
\hline
\end{tabular}

Tabela 12. De que forma a presença do CD em um ambiente hospitalar pode ser benéfica

\begin{tabular}{ccc}
\hline \multicolumn{3}{c}{$\begin{array}{c}\text { De que maneira a presença do cirurgião-dentista em um hospital } \\
\text { poderia ser benéfica? }\end{array}$} \\
\hline Assertiva & $\mathrm{N}^{\circ}$ (número) & $\%$ (por cento) \\
\hline No tratamento de traumas faciais & 198 & $23,18 \%$ \\
No diagnostico de lesões bucais & 183 & $21,43 \%$ \\
No atendimento de emergências odontológicas & 166 & $19,43 \%$ \\
Na capacitação de profissionais para higienização & 159 & $18,62 \%$ \\
bucal dos pacientes internados & 148 & $17,34 \%$ \\
Realizando procedimentos de higiene bucal & 854 & $100 \%$ \\
\hline
\end{tabular}

DISCUSSÃO

Araújo et al. $^{12}$ afirmam que a manutenção da higiene bucal de pacientes internados busca controlar o desenvolvimento de um biofilme de maior patogenicidade nos sítios da cavidade bucal, visto que, esses pacientes frequentemente encontram-se com 0 sistema imunológico debilitado e uma alteração de origem bacteriana aumentaria o risco de complicações sistêmicas. Wayama et al. ${ }^{10}$ acrescentam que a presença de um cirurgiãodentista no contexto hospitalar torna-se necessário para a realização de atividades curativas, preventivas e educativas em saúde bucal, buscando prevenir o desenvolvimento de problemas bucais comuns que, devido ao sistema imunológico debilitado do paciente, podem levar à piora do quadro clínico geral do indivíduo.

Os resultados obtidos por meio desse estudo apontam que a presença de um cirurgião-dentista na equipe multiprofissional do hospital, na percepção dos acadêmicos de odontologia da UFCG, é importante na prevenção de infecções $(54,65 \%)$ e na manutenção da saúde da cavidade bucal dos pacientes $(41,86 \%)$.

A grande maioria dos estudantes $(96,9 \%)$ que participaram da pesquisa também acredita que a presença de um cirurgiãodentista na equipe multiprofissional pode contribuir para melhorar o quadro clínico geral dos pacientes hospitalizados.

As informações obtidas corroboram com um estudo similar publicado por Amaral et al. ${ }^{13}$ com um grupo de cirurgiões-dentistas que não atuavam no serviço hospitalar, onde $96 \%$ desses profissionais concordaram que é importante a atuação deste profissional no acompanhamento de pacientes hospitalizados em UTI. Ainda nesse estudo, 55\% dos membros da equipe multiprofissional de UTI concordaram que haveria melhora do quadro clínico geral dos pacientes internados caso um cirurgião-dentista fosse incorporado a equipe.

De acordo com Deriso et al. $^{14} \mathrm{e}$ Yoneyama et al. $^{15}$, o tratamento odontológico colabora significativamente com a prevenção e/ou melhora da condição sistêmica, principalmente em paciente hospitalizado.

Godoi et al. ${ }^{16}$ e Kahn et al. ${ }^{17}$ afirmam que é possível perceber relevantes sinais de recuperação precoce quando 0 paciente hospitalizado recebe assistência odontológico, sendo assim, o cirurgião-dentista deve estar preparado para realizar sua atividade em ambiente diferenciado, visando proporcionar ao paciente melhores condições de saúde. Porém, os resultados obtidos nesse estudo demonstram que $(70,15 \%)$ dos estudantes não se sentem capacitados para atuar no âmbito hospitalar.

Segundo Vilella et al. ${ }^{18}$, a Odontologia em âmbito hospitalar possui grande importância, para os alunos de graduação em odontologia, uma vez que possibilita o contato com diversas situações que envolvem o tratamento multidisciplinar realizado por cirurgiõesdentistas, médicos e enfermeiros.

Os dados do presente trabalho apontam que $92,24 \%$ dos alunos de Odontologia da UFCG nunca tiveram uma experiência odontológica no âmbito hospitalar e que apenas $2,33 \%$ dos alunos não acham necessária essa experiência.

$\mathrm{Na}$ opinião dos alunos que participaram do presente estudo, o conteúdo referente à Odontologia Hospitalar é carente no currículo da graduação apesar de já terem ouvido falar sobre o assunto por docentes (42,63\%) e encontra-se, muitas vezes, abordado de forma fragmentada em outras disciplinas (12,40\%). Resultados parecidos foram obtidos por Wayama et al. ${ }^{10}$,demonstrando que $64 \%$ dos cirurgiõesdentistas participantes do seu estudo não tiveram o conteúdo durante sua formação acadêmica ou que esse assunto foi abordado de forma pouca significativa, comprovando que existem falhas na formação acadêmica do aluno de graduação, referente ao conteúdo de Odontologia Hospitalar.

$$
\text { Em relação aos componentes }
$$
curriculares que devem abordar tal conteúdo, a Traumatologia (16,67\%), a CBMF (15,54\%) e a Saúde coletiva e Odontologia preventiva $(11,90 \%)$ foram as disciplinas mais lembradas pelos acadêmicos. Alguns estudantes (10,53\%) ainda sugeriram que o conteúdo fosse abordado em uma disciplina eletiva. A CTBMF foi a especialidade odontologia mais lembrada pelos 
acadêmicos quando questionados sobre qual especialidade estaria mais capacitada para atuar no ambiente hospitalar (32,27\%).

Esses resultados são reflexos da recente legitimação da Odontologia Hospitalar no Brasil que ocorreu em 2004 com a criação da Associação Brasileira de Odontologia Hospitalar. Antes, o atendimento odontológico nos hospitais estava restrito à especialidade de Cirurgia e Traumatologia Bucomaxilofacial ou a execução de alguns procedimentos odontológicos que necessitavam de anestesia geral $^{2,19}$.

$\mathrm{Na}$ realidade odontológica de formação acadêmica e de pós-graduação brasileira, Miranda e Montenegro ${ }^{20}$ afirmam que pouco se transmite a respeito do atendimento odontológico a pacientes especiais, geriátricos, em UTI e suas peculiaridades, ou seja, as atividades de prevenção e curativa para pacientes impossibilitados de irem ao consultório odontológico são constantemente negligenciadas, deixando o paciente no poder de profissionais bem intencionados, mas que geralmente não dispõem de conhecimento específico e adequado.

De acordo com Lima et al. $^{21}$, a Odontologia vivência uma era holística em que o paciente deve ser visto como um todo, avaliando não apenas a cavidade bucal, mas seu estado de saúde geral, que pode estar comprometido pelo despreparo de profissionais em enfrentar determinadas situações no âmbito hospitalar. Portanto, existe a necessidade que durante a formação acadêmica o aluno seja incentivado e preparado para lidar com situações diferentes das vivenciadas diariamente em consultório.

Sobre o que os graduandos esperariam caso o conteúdo sobre a odontologia hospitalar fosse incluída no currículo do curso, segundo nosso estudo, (45,35\%) responderam estar capacitado para atuar em um hospital quando estivesse formado clínico geral. E saber como um cirurgião-dentista poderia atuar em um hospital e como deveria se comportar foi a resposta de $(20,55 \%)$ dos participantes. Um estudo semelhante realizado por Ferreira et al. ${ }^{22}$ apontou que $(51,25 \%)$ dos graduandos desejavam saber como deveria ser a postura, atuação e comportamento do cirurgião-dentista no hospital e outros $(46,25 \%)$ gostariam poder entrar e no mínimo atuar em um atendimento odontológico como acadêmico.

Wayama et al. $^{10}$ afirmaram que é de suma importância para o aluno de graduação, como um futuro cirurgião-dentista, ser incentivado e preparado durante a sua formação acadêmica para manejo do paciente em âmbito hospitalar. Ressaltaram ainda, a importância das faculdades de Odontologia abordarem este tema na grade curricular durante a graduação.

Saldanha et al. ${ }^{23}$ ressalvam a importância da formação/capacitação do cirurgião-dentista que presta atendimento hospitalar para que contribua efetivamente na prevenção da piora ou mesmo do desenvolvimento de problemas sistêmicos dos pacientes.

Lucas et al. ${ }^{24}$ realizaram um estudo sobre o ensino da Odontologia Hospitalar no sul do Brasil, foram avaliadas 40 instituições de ensino superior,onde apenas 6 instituições,sendo as mesmas da rede privada, apresentaram o componente curricular de Odontologia Hospitalar em seu Projeto Pedagógico do curso( PPC).

Conforme afirmam Scannapieco et al. ${ }^{25}$, aquele que pretende atuar no ambiente hospitalar como clínico geral, deve realizar um curso de habilitação com no mínimo 350 horas, sendo $30 \%$ de aulas práticas e $70 \%$ de aulas teóricas, podendo requerer após o término do curso, seu registro no Conselho Federal e Regional de Odontologia como habilitado em Odontologia Hospitalar.

CONCLUSÃO

A partir dos resultados desse trabalho, pode-se constatar que os graduandos do curso de odontologia da Universidade Federal de Campina Grande-UFCG, tem ciência da importância da odontologia hospitalar, porém, assumem não ter um bom conhecimento sobre o assunto e que não se sentem capacitados para atuar no ambiente hospitalar, sendo visível perceber que a maioria dos alunos ainda associa 0 atendimento hospitalar somente ao cirurgião bucomaxilofacial. Por esse motivo é indispensável que durante a formação acadêmica, sejam proporcionadas aos alunos, vivências que promovam a discussão criticoreflexiva sobre esta possibilidade de atividade profissional que esta em expansão.

REFERÊNCIAS

1. Brasil. Conselho federal de Odontologia. Código de ética odontológico. Disponível em:http://www.crosp.org.br/uploads/etica/6ac4d 2e1ab8cf02b189238519d74fd45.pdfAcessado em: 24 abril 2019

2. Aranega AM. Qual a importância da Odontologia Hospitalar? Rev Bras Odontol. 2012;69(1):90-3.

3. 3-Brasil. Projeto de lei no 2776/2008, de 13 de Fevereiro de 2008. Estabelece a obrigatoriedade da presença de profissionais 
de odontologia nas unidades hospitalares e dá outras providências. Rio de Janeiro; 2008.

4. Gaetti-jardim E, Setti JS, Cheade MFM, Mendonça JCG. Atencao odontológica a pacientes hospitalizados: revisão da literatura e proposta de protocolo de higiene oral. Rev bras cienc saude. 2013;11(35):31-6.

5. Pinheiro TS, Almeida TFA saúde bucal em pacientes de UTI. Rev Bahiana Odontol. 2014;5(2):94-103.

6. Toledo GB, Da Crurz ICF. The importance of the oral hygiene in Intensive Care Unit as a way of prevention of nosocomial infection-Sistematic Literature Review. J Spec Nurs Care. 2009; 2(1).

7. Walsh MC, Kim N, Kadono Y, Rho J, Lee S Y, Lorenzo J, Choi AY. Osteoimmunology: interplay between the immune system and bone metabolism. Ann Rev Immunol. 2006;24(1): 33-63

8. Aaa JA, Barbuto SM, Alpagot T, Olsen I, Dewhirst FE, Paster BJ. Subgingival plaque microbiota inHIV positive patients. J Clin Periodontol. 2007;34(3):189-95.

9. Azarpazhooh A, Leake JL.Systematic review of the association between respiratory diseases and oral health. J Periodontol. 2006;77(9): 1465-82.

10. Wayma MT, Aranega AM, Bassi APF, Ponzoni D, Garcia Junior IR . Grau de conhecimento dos cirurgiões-dentistas sobre a Odontologia Hospitalar. Rev. Bras. Odontol. 2014;71(1): 48-52.

11. Jordão TF. Conhecimento de acadêmicos de odontologia sobre a atuação do cirurgiãodentista no contexto hospitalar [monografia]. Universidade Estadual da Paraíba (UEPB), Araruna - PB; 2016

12. Araújo RJG, Oliveira LCG, Hanna LMO, Correa AM, Carvalho LHV, Alvares NCSF. Análise de percepções e ações de cuidados bucais realizados por equipes de enfermagem em unidades de tratamento intensivo. Rev Bras Ter Intensiva. 2009;21(1):38-44.

13. Amaral COF, Marques JA, Bovolato MC, Parizi AGS, Oliveira A, Straioto FGI. Importância do cirurgião-dentista em Unidade de Terapia Intensiva: avaliação multidisciplinar. Rev Assoc Paul Cir Dent. 2013;67(2):107-11.

14. Deriso AJ, Ladowski JS, Dillon TA, Justice JW, Peterson AC. Chlorhexidinegluconate $0.12 \%$ oral rinse reduces the incidence of total nosocomial respiratory infection and nonprophylactic system antibiotic use in patients undergoing heart surgery. Chest. 1996; 109:1556-61

15. Yoneyama T, Yoshida M, Ohrui T, Mukaiyama $\mathrm{H}$, Okamoto $\mathrm{H}$, Hoshiba $\mathrm{K}$ et al. Oral Care Working Group. Oral care reduces pneumonia in older patients in nursing homes. J Am Geriatr Soc. 2002;50(3):430-33.

16. Godoi APT, Francesco AR, Duarte A, Kemp APT ,Silva-Lovato $\mathrm{CH}$. Odontologia hospitalar no Brasil. Uma visão geral. Rev Odontol Unesp. 2009;38(2):105-9.

17. Kahn S, Garcia $\mathrm{CH}$, Galan Júnior J, Namen FM, Machado WAS, Silva Júnior JA et al. Avaliação da existência de controle de infecção oral nospacientes internados em hospitais do estado do Rio de Janeiro. Cienc saúde coletiva.2008;6(13):1825-31.

18. Vilella FMS, Parras AA, Ferreira AR, Ramires GAD, Silva NM, Botacin PR et al. O estágio do ambiente hospitalar como eficiente experiência para o ensino, a pesquisa e a extensão dos alunos do curso de odontologia. Rev Ciência Extensão. 2011;7(3):51.

19. Aguiar ASW, Guimarães MV, Moraes RMP, Saraiva JLA. Atenção em saúde bucal em nível hospitalar: relato de experiência de integração ensino/serviço em Odontologia. Extensio. 2010; 7(9):100-10.

20. Miranda AF, Montenegro FL. O cirurgiãodentista como parte integrante de uma equipe multidisciplinar no atendimento aos idosos. Rev Paul Odontol. 2009;31(3):15-9.

21. Lima, DC, Saliba NA, Garbin AJ, Fernandes LA, Garbin CA. A importânciada saúde bucal na ótica de pacientes hospitalizados. Ciênc saúde coletiva. 2011;16(1):1173-80.

22. Ferreira LDS, Ribeiro ESA, Dos Santos REA. Conhecimento dos acadêmicos de odontologia da UEA sobre a odontologia hospitalar. RvAcBO. 2017.26(1):38-43.

23. Saldanha KDF, Costa DC, Peres PI, Oliveira MM, Masocatto DC, Gaetti Jardim EC. A Odontologia Hospitalar: revisão. Arch Health Invest. 2015;4(1):58-68.

24. Lucas BB, Vieira Júnior JLR, Besegato JF, Caldarelli PG. Ensino da Odontologia Hospitalar no sul do Brasil. Rev ABENO. 2017;17(2):68-75.

25. Scannapieco F. Atuação em ambiente hospitalar exige dos Cirurgioes-Dentistas conhecimentos específicos e evidencia a importância do trabalho multidisciplinar e interprofissional. Rev Assoc Paul Cir Dent. $2011 ; 65(5): 323$ 


\section{CONFLITO DE INTERESSES}

Os autores declaram não haver conflitos de interesse

AUTOR PARA CORRESPONDÊNCIA

\section{José Henrique de Araújo Cruz}

Rua Paulo Diogenes, número 57, bairro Centro

59990-000 Rafael Fernandes - RN, Brasil

Telefone: (83) 99625-0125.

E-mail: henrique_araujo1992@hotmail.com

Submetido em 07/04/2020

Aceito em 23/10/2020 